Editorial

\title{
Acknowledgement to Reviewers of Universe in 2018
}

\author{
Universe Editorial Office \\ MDPI, St. Alban-Anlage 66, 4052 Basel, Switzerland \\ Published: 11 January 2019
}

\begin{abstract}
Rigorous peer-review is the corner-stone of high-quality academic publishing. The editorial team greatly appreciates the reviewers who contributed their knowledge and expertise to the journal's editorial process over the past 12 months. In 2018, a total of 154 papers were published in the journal, with a median time to first decision of 22 days, and a median time to publication of 45.5 days. The editors would like to express their sincere gratitude to the following reviewers for their cooperation and dedication in 2018:
\end{abstract}

\begin{tabular}{|c|c|}
\hline Aarts, Gert & Blas, Diego \\
\hline Abreu, Everton M.C. & Blaschke, David \\
\hline Adams, Jenni & Blázquez-Salcedo, Jose Luis \\
\hline Aichelburg, Peter & Blümlein, Johannes \\
\hline Alexander, Stephon & Bobev, Nikolay \\
\hline Andrew, Keith & Bodendorfer, Norbert \\
\hline Antoniadis, Ignatios & Bojowald, Martin \\
\hline Arbuzov, Andrej & Bondar, Denys I. \\
\hline Aros, Rodrigo & Bonga, Beatrice \\
\hline Arraut, Ivan & Bopp, Fritz W. \\
\hline Astrakharchik, Gregory & Böröczky, Károly \\
\hline Avrorin, Alexander & Borowiec, Andrzej \\
\hline Balabanski, Dimiter L. & Boulanger, Nicolas \\
\hline Balakin, Alexander B. & Bozapalidis, Symeon \\
\hline Bamba, Kazuharu & Braden, Jonathan \\
\hline Bamba, Kazuharu & Brandenberger, Robert H. \\
\hline Bartelmann, Matthias & Bravina, Larissa \\
\hline Baryshev, Yurij & Brodsky, Stanley J. \\
\hline Bass, Steven D. & Bronnikov, Kirill \\
\hline Beccaria, Matteo & Brown, Ethan \\
\hline Beccaria, Matteo & Buchbinder, Joseph L. \\
\hline Bejger, Michał & Bulnes, Franc \\
\hline Bekaert, Xavier & Burdin, Sergey \\
\hline Belli, Pierluigi & Burgio, Fiorella \\
\hline Berezhiani, Zurab & Burko, Lior \\
\hline Bernabei, Rita & Cacciatori, Sergio Luigi \\
\hline Bernabeu, Jose & Cai, Yi-Fu \\
\hline Bhattacharya, Tanmoy & Calcagni, Gianluca \\
\hline Biermann, P. L. & Caravelli, Francesco \\
\hline
\end{tabular}


Carballo-Rubio, Raúl

Cariglia, Marco

Carinena, Jose F.

Carmona, Jose Manuel

Casadio, Roberto

Catenacci, Roberto

Celis, Alejandro

Cembranos, Jose

Cerri, Alessandro

Chamel, Nicolas

Chamoun, N.

Chen, Chuan-Hung

Chepel, Vitaly

Cherdack, Daniel

Chervon, Sergei

Choi, Suyong

Christian, Codra

Cleave, Gerald

Coelho, João A.B.

Cohen, Thomas

Combrinck, Ludwig

Costa Ulhoa, Srgio

Cotsakis, Spiros

Covi, Laura

Craig, Nathaniel

Csanád, Máté

Csörgő, Tamás

Dahal, Rajendra P

D'Ambrosio, Giancarlo

Danehkar, Ashkbiz

Dappiaggi, Claudio

Das, Saurya

Dasgupta, Keshav

David, Edwin Alvarez Castillo

De Lucas, Javier

De Martino, Ivan

Del Popolo, A.

Del Sordo, Fabio

Deppman, Airton

Deramo, Francesco

Dexheimer, Veronica

Di Giacomo, Adriano

Di Marco, Alessandro
Dirkx, Dominic

Doi, Takahiro

Dokuchaev, Vyacheslav

Ivanovich

Duhr, Claude

Eleuch, Hichem

Emelyanov, Viacheslav

Escamilla Herrera, Lenin

Francisco

Evans, Justin

Fabris, Júlio C.

Fabris, Júlio César

Fadin, Victor

Faraggi, Alon

Feix, Martin

Feoli, Antonio

Fernando, Sharmanthie

Ferrer, Francesc

Ferrera, Giancarlo

Fischer, Tobias

Fletcher, Samuel C.

Flores, José Luis

Fortin, Morgane

Frassino, Antonia M.

Fredenhagen, Stefan

Friedman, Yaakov

Friesen, Alexandra

Furukawa, Kazuro

Gai, Moshe

Gaitanos, Theodoros

García, J Antonio

Garzelli, Maria Vittoria

Gasperini, Maurizio

Gavrilik, Alexandre

Gazeau, Jean-Pierre

Gazis, Evangelos

Gerosa, Davide

Ghiglieri, Jacopo

Giedt, Joel

Giocoli, Carlo

Girelli, Florian

Giunti, C.

Glaser, Christian 
Glorioso, Paolo

Gorbenko, Victor

Gourgouliatos, Konstantinos

Grassi, Pietro Antonio

Grøn, Øyvind

Gubitosi, Giulia

Guendelman, Eduardo

Guendelman, Eduardo L.

Hackmann, Eva

Halliwell, Jonathan

Hamber, Herbert W.

Hammad, Fayçal

Harko, Tiberiu

Hatfield, Peter

Hatta, Yoshitaka

Hees, Aurelien

Helayël-Neto, José Abdalla

Hererra, Luis

Hessels, J.W.T

Higinbotham, Douglas W.

Hikida, Yasuaki

Hofmann, Ralf

Horváthy, Péter A.

Huang, Xu-Guang

Huber, Markus

Hundertmark, Markus

Ibarra, Alejandro

Iorio, Lorenzo

Iorio, Lorenzo

Ivanov, Igor

Ivanov, Yu. B.

Jaikumar, Prashanth

Jassal, Harvinder

Jegerlehner, Fred

Jones, Benjamin

Joyce, Michael

Jurčišinová, Eva

Jurić, Tajron

Kajantie, Keijo

Kaminker, Alexander D.

Karsch, Frithjof

Kashi, Amit

Kevin, Stovall
Khurshudyan, Martiros

Kibler, Maurice

Kibler, Maurice R.

Kim, Sung-Won

Kimura, Rampei

Kirillov, Alexander

Kleidis, Kostas

Koivisto, Tomi

Koivisto, Tomi

Kokkotas, Kostas

Konkowski, Deborah A.

Koshelev, Alexey S.

Krajewski, Thomas

Krämer, Manuel

Krämer, Manuel

Krasnov, Kirill

Kuznetsova, Zhanna

Gennadyevna

L'Huillier, Benjamin

Lambiase, Gaetano

Lazar, Marian

Lecian, Orchidea Maria

Lee, Chung-Chi

Lehner, Luis

Leontaris, George K.

Lepe, Samuel

Li, Gang

Li, Liang

Li, Shirley Weishi

Li, Yang

Li, Yu-Feng

Liberali, Valentino

Licata, Ignazio

Liss, Tony M.

Liu, Fuhu

Liu, Fu-Hu

Livine, Etera

Loebbert, Florian

Logoteta, Domenico

López Corredoira, Martín

Lorca, David

LoSecco, John M.

Lunin, Oleg 


\begin{tabular}{|c|c|}
\hline Luongo, Orlando & Olmedo, Javier \\
\hline Macedo, Caio & Olmedo, Javier \\
\hline Makhlouf, Abdennacer & Olmo, Gonzalo J. \\
\hline Malafarina, Daniele & Ong, Yen Chin \\
\hline Malka, Dror & Oriti, Daniele \\
\hline Mao, Pujian & Ovgun, Ali \\
\hline Mariani, Camillo & Pagliara, Giuseppe \\
\hline Martino, Ivan De & Pal, Bilas K. \\
\hline Matsumoto, Jiro & Palla, Laszlo \\
\hline Mavromatos, Nikolaos & Papadopoulos, Basil \\
\hline McNeile, Craig & Partouche, Hervé \\
\hline Mehlhase, Sascha & Pasechnik, Roman \\
\hline Mendoza, Sergio & Pengming, Zhang \\
\hline Merlo, Vittorio & Percacci, Roberto \\
\hline Michel, Florent & Peter, Patrick \\
\hline Mielke, Eckehard W. & Petrov, Albert \\
\hline Millington, Peter & Pfeiffer, Harald \\
\hline Milton, Kimball A. & Picanço, Rodrigo \\
\hline Miramonti, Lino & Pinfold, James \\
\hline Mirshekari, Saeed & Polyakov, M. V. \\
\hline Mitsou, Vasiliki & Pradhan, Anirudh \\
\hline Modanese, Giovanni & Procureur, Sébastien \\
\hline Monthus, Cecile & Profumo, Stefano \\
\hline Morgano, Manuel & Providência, Constança \\
\hline Moustakidis, Charalampos & Pullin, Jorge \\
\hline Mück, Wolfgang & Queiroz, Farinaldo \\
\hline Nakonieczny, Łukasz & Quevedo, Hernando \\
\hline Nara, Yasushi & Radi, Amr \\
\hline Nardelli, Giuseppe & Rahvar, Sohrab \\
\hline Nathanail, Antonios & Rassias, Themistocles M. \\
\hline Nielsen, Marina & Ratti, Claudia \\
\hline Nomura, Takaaki & Reece, Matt \\
\hline Nomura, Yasunori & Reinhardt, Hugo \\
\hline Novák, Tamás & Rimmer, Paul \\
\hline Nozari, Kurosh & Rinaldi, Massimiliano \\
\hline Nozzoli, Francesco & Rizos, John \\
\hline Nucita, Achille A. & Roberts, Benjamin \\
\hline Odintsov, Sergei & Roberts, Craig D. \\
\hline Odintsov, Sergei & Robles-Perez, Salvador \\
\hline Ohme, Frank & Rodriguez, Leo \\
\hline Oikonomou, Vasileios & Rogatko, Marek \\
\hline Oldofredi, Andrea & Romatschke, Paul \\
\hline Oliveira, Leandro A. & Röpke, Gerd \\
\hline
\end{tabular}


Rovelli, Carlo

Roy, Shibaji

Rozas-Fernandez, Alberto

Saal, Margus

Sahoo, Pradyumn Kumar

Saikawa, Kenichi

Saikawa, Kenichi

Sakellariadou, Mairi

Salvioni, Ennio

Saridakis, Emmanuel N.

Sarlis, Nicholas V

Sasaki, Chihiro

Saueressig, Frank

Scampoli, Paola

Schaefer, Thomas

Schenke, Bjoern

Schenkel, Alexander

Schmitt, Andreas

Schroeder, Frank

Schweitzer, Peter

Sedrakian, Armen

Senjanovic, Goran

Shaikh, Rajibul

Shao, Lijing

Shao, Lijing

Shen, Chun

Shen, Chun

Sheykhi, Ahmad

Shimmin, Chase

Shtanov, Yuri

Shyam, Vasudev

Siegert, Thomas

Siklér, Ferenc

Silva, Hector O.

Singleton, Douglas

Sitarz, Andrzej

Smirnov, Alexei

Spagnolo, Nicolò

Spallicci, Alessandro

Speziale, Simone

Spira, Michael

Stöcker, Horst

Stuchlík, Zdeněk
Šumbera, Michal

Sun, Jia Rui

Tanahashi, Norihiro

Tawfik, Abdel Nasser

Thompson, Lee

Torrielli, Alessandro

Torrieri, Giorgio

Tortola, Mariam

Troessaert, Cedric

Tronconi, Alessandro

Tsukamoto, Naoki

Tsupko, Oleg

Turko, Ludwik

Valenzuela, Mauricio

Velten, Hermano

Venanzoni, Graziano

Vento, Vicente

Vergara, J. David

Viel, Matteo

Vilja, Iiro

Visinelli, Luca

Vittino, Andrea

Wahlberg, Hernan

Wang, Donggang

Wang, Michael H.L.S.

Wang, Sai

Weber, Fridolin

Wei, Hanyu

West, Peter

Wieland, Wolfgang

Wiggins, Brandom

Wildberger, N.J.

Wimberger, Sandro

Wlodarczyk, Zbigniew

Woodard, Richard

$\mathrm{Xu}$, Donglian

$\mathrm{Xu}$, Guochang

$\mathrm{Xu}$, Jun

$\mathrm{Xu}$, Renxin

Yamada, Toshifumi

Yeom, Dong-han

Zabrodin, Evgeny

Zaccolo, Valentina 
Zaninetti, Lorenzo

Zapata, José Antonio

Zerwekh, Alfonso

Zhang, Tong-jie

Zhidenko, Alexander
Zimdahl, Winfried

Zimdahl, Winfried

Zinoviev, Yurii

Zipfel, Antonia

(). 1

(C) 2019 by the authors. Licensee MDPI, Basel, Switzerland. This article is an open access article distributed under the terms and conditions of the Creative Commons Attribution (CC BY) license (http://creativecommons.org/licenses/by/4.0/). 